\title{
Editorial
}

\section{A Renewed Question in Haemodialysis Patients: Should We Correct Low Serum Magnesium Levels?}

\author{
Mario Cozzolino \\ Department of Health Sciences, Renal Division, San Paolo Hospital, University of Milan, Milan, Italy
}

\section{Key Words}

Magnesium · CKD-MBD

Disturbances in magnesium $(\mathrm{Mg})$ metabolism are not so rare in chronic kidney disease (CKD) and dialysis patients, especially in those affected by diabetes, insulin resistance, inflammation, vascular calcification (VC), and higher mortality [1]. Furthermore, renal patients are prone to higher cardiovascular (CV) morbidity and mortality. CV disease in CKD is associated with VC and mineral bone disorders, which are regulated by hormone disturbances (FGF23/klotho-PTH-Vitamin D axis) [2]. Recently, an independent association between low Mg levels and $\mathrm{CV}$ disease in dialysis patients has been observed, resuscitating this ion as a renewed and active player in vascular aging.

Consistent results from both pre-clinical and clinical research showed that CV susceptibility in CKD is strongly associated with a considerable acceleration of vascular aging in the context of the chronic kidney disease-mineral bone disorder syndrome (CKD-MBD), in which new biomarkers are needed to improve the understanding toward further intervention strategies [3].

In the present issue of the Blood Purification, Matias et al. show the association between low serum $\mathrm{Mg}$ levels and increased CV risk factor in 206 haemodialysis (HD) pa- tients [4]. This elegant observational analysis illustrates that patients with higher pulse pressure (PP) $(\geq 65 \mathrm{~mm}$ $\mathrm{Hg})(\mathrm{p}=0.01)$, left ventricular hypertrophy (LVMI $\geq 125$ $\left.\mathrm{g} / \mathrm{m}^{2}\right)(\mathrm{p}=0.02)$, and higher VC (SVCS $\left.\geq 3\right)(\mathrm{p}=0.008)$ have significantly lower serum $\mathrm{Mg}$ concentrations.

In addition, Kaplan-Meier analysis shows that both all-cause and CV mortality were associated with a significant lower 48-month survival in HD patients with the level of serum $\mathrm{Mg}$ being lower than $1.15 \mathrm{mmol} / \mathrm{l}$, thereby assigning $\mathrm{Mg}$ a role of a 'renewed' independent negative predictor of mortality.

A growing body of evidence is suggesting a potential role for $\mathrm{Mg}$ in different diseases such as CKD-MBD, diabetes, low insulin resistance, poor endothelial reactivity, higher oxidative stress, increased intima-media thickness, vascular calcification, progression of CKD, and mortality [5].

Nevertheless, the maintenance of adequate intracellular Mg levels appears essential for life per se, considering that $\mathrm{Mg}$ is a fundamental co-enzyme of vital biochemical reactions just like Krebs cycle and glycolysis and it is essential for the maintenance of RNA and DNA stability. Thus, Mg may represent a cardinal biomarker and regulator of vascular aging in humans, with special emphasis in CKD patients. Furthermore, nephrologists are called to a growing comprehension of $\mathrm{Mg}$ physiology as a consequence of the renewed adoption of $\mathrm{Mg}$ as a phosphate

\section{KARGER}

E-Mail karger@karger.com

www.karger.com/bpu
(C) 2015 S. Karger AG, Basel

$0253-5068 / 15 / 0384-0242 \$ 39.50 / 0$
Mario Cozzolino, MD, PhD, FERA

Renal Division and Laboratory of Experimental Nephrology, Dipartimento di Scienze della Salute, Università di Milano, Renal Division, S. Paolo Hospital

Via A. di Rudinì, 8, IT-20142 Milan (Italy)

E-Mail mario.cozzolino@unimi.it 
binder. Data by Matias et al. [4] confirm the growing interest of the nephrology community pointing toward $\mathrm{Mg}$ metabolism with particular emphasis on CKD-MBD patients.

In summary, it can be argued that hypomagnesemia or, even more strikingly, lower Mg levels may be actively detrimental for CKD-MBD in $\mathrm{HD}$ patients, contributing to low bone turnover, poor insulin sensitivity and lower protection against inflammation and oxidative stress in arteries leading to higher VC. This study by Matias et al. [4] signals a relevant step forward in our epidemiological knowledge about the important role played by $\mathrm{Mg}$ in the arena of vascular aging in CKD-MBD. However, further investigations are required to better elucidate whether $\mathrm{Mg}$ supplementation may improve vascular aging in HD patients and to identify the optimal serum $\mathrm{Mg}$ levels in this population.

Despite all that we learned, learn, and will learn about the role of $\mathrm{Mg}$ on $\mathrm{CV}$ disease in CKD patients, we have been left only with a hypothetical framework about how to treat patients with lower serum Mg levels. A putative 'final' answer should be tested with a global-multiple therapeutic approach to treat simultaneously several components of CKD-MBD, through a well-designed, new randomised controlled trial.

\section{References}

1 Galassi A, Cozzolino M: Magnesium: a renewed player of vascular ageing in diabetic CKD patients? Clin Kidney J 2014, Epub ahead of print.

$\checkmark 2$ Ronco C, Cozzolino M: Mineral metabolism abnormalities and vitamin $\mathrm{D}$ receptor activation in cardiorenal syndromes. Heart Fail Rev 2012;17:211-220
Cozzolino M, Ureña-Torres P, Vervloet MG, Brandenburg V, Bover J, Goldsmith D, Larsson TE, Massy ZA, Mazzaferro S; on Behalf of the CKD-MBD Working Group of ERAEDTA: Is chronic kidney disease-mineral bone disorder (CKD-MBD) really a syndrome? Nephrol Dial Transplant 2014, Epub ahead of print.
4 Matias P, Mendes M, et al: Lower serum magnesium is associated with cardiovascular risk factors and mortality in haemodialysis patients. Blood Purif 2014;38:244-252.

5 Sakaguchi Y, Fujii N, Shoji T, et al: Hypomagnesemia is a significant predictor of cardiovascular and non-cardiovascular mortality in patients undergoing hemodialysis. Kidney Int 2013;85:174-181. 